\title{
Evidence summary: does 'routine' scaling have any beneficial effects, or is it a waste of time?
}

\author{
Original question submitted by P. C. W. Chambers, March 2010 \\ Reviewer: Chris Fox' \\ Information Scientists: Helen Nield* and Anne Littlewood**
}

KEY TERMS

- Scaling: removal of plaque, calculus, debris and staining from crown and root surfaces.

- Polish: mechanical removal of residual extrinsic stains and deposits.

- Routine: either providing an intervention at regular intervals to patients or as a matter of routine (ie regardless of assessed need for treatment or prevention).

- Effectiveness: measurable sustained benefits in periodontal or overall oral health.

- Cost-effectiveness: costs to patient and practitioner compared with benefits arising from treatment received.

Since August 2009, members of the Primary Care Dentistry Research Forum (www.dentistryresearch.org) have taken part in an online vote to identify questions in day-to-day practice that they felt most needed to be answered with conclusive research. The question which received the most votes formed the subject of a critical appraisal of the relevant literature. Each month a new round of voting takes place to decide which further questions will be reviewed. Dental practitioners and dental care professionals are encouraged to take part in the voting and submit their own questions to be included in the vote by joining the website.

This paper details a summary of the findings of the sixth critical appraisal. In conclusion, the critical appraisal identified a high quality systematic review carried out in 2007 relating to routine scaling. Since the Cochrane Review of 2007, we could find no new randomised controlled trials to assess the beneficial and sustained effects of routine scaling and polishing.

\section{BACKGROUND}

In 2008/9, scale and polish was the most common adult clinical dental treatment in England, being included in almost three million courses of treatment. ${ }^{1}$ However, scale and polish treatments for periodontal health have previously been described as ineffective procedures, ${ }^{2}$ so what is the evidence to support such activity?

\section{REVIEW METHOD}

An initial search was made of Ovid MEDLINE (1950 to week 3, February 2010) using the search terms dental scaling, routine, treatment outcome, effectiveness, and pros or cons. Eightysix papers were identified and 85 papers excluded. One Cochrane review (2007

\footnotetext{
${ }^{1}$ PhD Student, Peninsula Dental School

*BDA Library Manager; ; Trials Search Coordinator and Feedback Editor, Cochrane Oral Health Group Correspondence to: Beth Caines, Shirley Glasstone Hughes Trust Fund Administrator, British Dental Association, 2 Caspian Point, Cardiff Bay, CF10 4D0 Email: b.caines@bda.org; Tel: +44 (0)2920436184 Sponsored by the Shirley Glasstone Hughes Trust
}

DOI: $10.1038 /$ sj.bdj.2010.451 update) on routine scale and polish for periodontal health in adults was identified and sourced. ${ }^{3}$

Because we found a relatively recent and high quality systematic review relevant to the review question, we then sought to identify any relevant studies published since the Cochrane review. Three of the four search strategies used in the Cochrane review were repeated for the period 2007-March 2010: the Cochrane Oral Health Group (COHG) Trials Register, the Cochrane Central Register of Controlled Trials (CENTRAL) and MEDLINE (OVID). See Appendices 1-3 of the Cochrane review for detailed search strategies.

- COHG Trials Register: 27 titles identified (2007 onwards), 25 excluded.

One paper in addition to the Cochrane review was accessed in full and examined; one paper rejected

- CENTRAL: 34 titles identified (2007 onwards), 33 titles other than the Cochrane review excluded

- MEDLINE (OVID): 97 titles identified, 96 titles other than the Cochrane review excluded.
Further contact was made with Paul Beirne, corresponding author of the Cochrane review.

\section{FINDINGS}

In total, 158 titles and abstracts arising from the three repeated Cochrane review searches were screened. One potentially relevant paper was retrieved as full text and reviewed. Ultimately no recent paper was found that met the inclusion criteria of the Cochrane review.

We could find no new randomised controlled trials to assess the beneficial and sustained effects of routine scaling and polishing. The conclusions of the Cochrane review remain current:

'The research evidence is of insufficient quality to reach any conclusions regarding the beneficial and adverse effects of routine scaling and polishing for periodontal health and regarding the effects of providing this intervention at different time intervals. High quality clinical trials are required to address the basic questions posed in this review.' 
Table 1 Summary of the Cochrane intervention review on routine scale and polish for periodontal health in adults (extracts/adapted from review/ review abstract)

\begin{tabular}{|c|c|}
\hline Main objective & $\begin{array}{l}\text { To determine the beneficial and harmful effects of routine scaling and polishing for periodontal health, and also at differ- } \\
\text { ent time intervals. }\end{array}$ \\
\hline Search strategy & COHG Trials Register, CENTRAL, MEDLINE and EMBASE. Most recent search 5 March 2007. \\
\hline Selection criteria & $\begin{array}{l}\text { Design - random allocation of participants } \\
\text { Participants - anyone with erupted permanent dentition judged to have had routine scale and polish } \\
\text { Interventions - routine scale and polish and also provided at different time intervals } \\
\text { Outcomes - tooth loss, plaque, calculus, gingivitis, bleeding and periodontal indices, changes in probing depth, attach- } \\
\text { ment change, patient-centred outcomes and economic outcomes. }\end{array}$ \\
\hline Main results: & Nine studies included. All studies assessed as having a high risk of bias. \\
\hline $\begin{array}{l}\text { 1. Comparison between scale and } \\
\text { polish and no scale and polish }\end{array}$ & $\begin{array}{l}\text { Two split-mouth studies: one involved patients attending a recall programme following periodontal treatment; no statisti- } \\
\text { cally significant differences for plaque, gingivitis, attachment loss between intervention and control groups at each time } \\
\text { point during the one year trial. The second study, involving adolescents in a developing country and having had no dental } \\
\text { care for at least five years, reported statistically significant differences in calculus and gingivitis scores between interven- } \\
\text { tion and control groups at } 6,12 \text { and } 22 \text { months following one scale and polish treatment. }\end{array}$ \\
\hline $\begin{array}{l}\text { 2. Comparisons between routine } \\
\text { scale and polish provided at } \\
\text { different time intervals }\end{array}$ & $\begin{array}{l}\text { Some statistically significant differences found in favour of scaling and polishing provided at more frequent inter- } \\
\text { vals: } 2 \text { weeks versus } 12 \text { months for outcomes of plaque, gingivitis, pocket depth, attachment change; } 3 \text { months versus } \\
12 \text { months for outcomes of plaque, calculus and gingivitis. }\end{array}$ \\
\hline \multicolumn{2}{|l|}{ Authors' conclusions: } \\
\hline 1. Implications for practice & $\begin{array}{l}\text { The research evidence is of insufficient quality and limited quantity to allow confident statements to be made regarding } \\
\text { the beneficial and harmful effects of routine scaling and polishing for periodontal health and regarding the frequency of } \\
\text { provision of this intervention to different patient populations. }\end{array}$ \\
\hline 2. Implications for research & $\begin{array}{l}\text { 2.1 Well conducted trials are needed in this area, with sufficient patient numbers, and of five or more years' duration } \\
\text { 2.2 Some trials should be undertaken in primary care settings } \\
\text { 2.3 Outcomes should include clinical measures and tooth loss, patient-centred factors and economic factors. May also } \\
\text { include caries outcomes } \\
\text { 2.4 Studies are needed to determine the clinical- and cost-effectiveness of routine scaling and polishing provided by dif- } \\
\text { ferent dental personnel } \\
\text { 2.5 In future studies to define what levels of outcome improvements are clinically significant. }\end{array}$ \\
\hline
\end{tabular}

It is understood that a further update of the Cochrane review may be undertaken in 2011. A summary of the review is given in Table 1.
1. The NHS Information Centre, Dental and Eye Care Team. Clinical dental report, England and Wales: quarter 3 and quarter 4,2008/9 - experimental statistics. London: The Health and Social Care Information Centre, 2010.

2. Sheiham A. Minimal intervention in dental care. Med Principles Pract 2002; 11(Suppl 1): 2-6 3. Beirne P V, Worthington H V, Clarkson J E. Routine scale and polish for periodontal health in adults. Cochrane Database Syst Rev 2007; (4): CD004625. DOI: 10.1002/14651858. CD004625.pub3. 